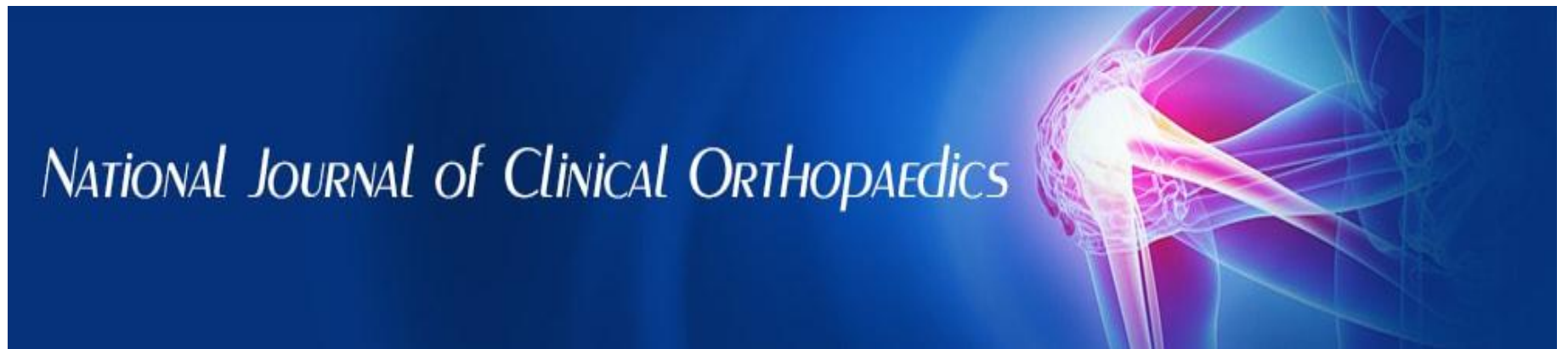

ISSN (P): 2521-3466

ISSN (E): 2521-3474

(C) Clinical Orthopaedics

www.orthoresearchjournal.com

$2020 ; 4(3): 12-14$

Received: 10-06-2020

Accepted: 11-07-2020

Dr. Sukumar Nayak

Associate Professor, Department of Physiotherapy, Garden City

College, Bengaluru, Karnataka,

India

Dr. Manjunatha A

Consultant Orthopedician,

Srinivasa Multispecialty

Hospital, Hoskote, Bengaluru,

Karnataka, India

Dr. Preethish Kumar Thakur PT

Consultant Physiotherapist,

Srinivasa Multispecialty

Hospital, Hoskote, Bengaluru,

Karnataka, India
Corresponding Author:

Dr. Manjunatha A

Consultant Orthopedician,

Srinivasa Multispecialty

Hospital, Hoskote, Bengaluru,

Karnataka, India

\section{Effect of elastic anti pronation taping technique along with Cryotherapy in decreasing pain and increasing ankle dorsiflexion range of motion in female subjects with medial tibia stress syndrome}

\author{
Dr. Sukumar Nayak, Dr. Manjunatha A and Dr. Preethish Kumar \\ Thakur PT
}

DOI: https://doi.org/10.33545/orthor.2020.v4.i3a.228

\section{Abstract}

MTSS is frequently seen, especially in running and jumping athletes and in military personal, currently it is thought that MTSS is a bony over load injury, i.e., the tibia bone bends during weight bearing activities causing strain in the tibia. This strain normally causes micro damage in the bone, which leads to bone adaptation processes to strengthen the bone to resist tibia bending. It has been estimated that this condition has accounted for $4 \%$ to $35 \%$ of overuse leg injuries in the athletic and military populations. Research has shown that MTSS in runners and military personal responsible for $13.2 \%$ to $17.3 \%$ of all running injuries and approximately $22 \%$ of injuries in aerobic dancer. Total of 60 subjects with age between 25 to 35 years, were assigned into two groups using convenience sampling method according to inclusion and exclusion criteria. In the case of any drop out during the study, a subject with same inclusion criteria was selected.

Keywords: Elastic Anti Pronation Taping Technique, Cryotherapy, Medial Tibia Stress Syndrome

\section{Introduction}

Medial Tibia stress syndrome is a debilitating overuse injury of the tibia sustained by individuals who run regularly on even or uneven surfaces being particularly affected, characterized by diffuse tibia antero-medial or posterior-medial surface subcutaneous periostitis ${ }^{[1]}$.

It has been estimated that this condition has accounted for $4 \%$ to $35 \%$ of overuse leg injuries in the athletic and military populations. Research has shown that MTSS in runners and military personal responsible for $13.2 \%$ to $17.3 \%$ of all running injuries and approximately $22 \%$ of injuries in aerobic dancer ${ }^{[2]}$.

A significant relationship existed between gender and medial tibia stress syndrome, with female are more likely to develop medial tibia stress syndrome than male, however only increased BMI and female gender were correlated with incidence of MTSS in six of the seven studies ${ }^{[3]}$. Yates et al found a significant relationship between the development of MTSS and female gender where female naval recruits were twice as likely to develop the condition as male recruits. Female demonstrated a $52.9 \%$ incidence rate as compared to the rate of $28 \%$ seen in males. A risk estimate revealed a relative risk of $2.03^{[4]}$.

MTSS is frequently seen, especially in running and jumping athletes and in military personal, currently it is thought that MTSS is a bony over load injury, i.e., the tibia bone bends during weight bearing activities causing strain in the tibia. This strain normally causes micro damage in the bone, which leads to bone adaptation processes to strengthen the bone to resist tibia bending. When this strain exceeds a certain threshold and becomes overloaded, the osteoclast activity may outpace osteoblast activity, leading to local tibia osteopenia ${ }^{[5]}$. Dysfunction of the tibiais posterior, tibiais anterior, and soleus muscles are commonly implicated ${ }^{[6]}$. 


\section{Methodology}

Total of 60 subjects with age between 25 to 35 years, wer assigned into two groups using convenience sampling method according to inclusion and exclusion criteria.

In the case of any drop out during the study, subjects with same inclusion criteria was selected.

\section{Inclusion Criteria}

- Female subjects of age between 25 to 30 years.

- Subjects who are having flat foot.(foot print)

- Subjects who are having pronated foot. (navicular drop test)

- Insidious onset of pain unrelated to any traumatic event.

- Subjects presenting the following signs.

a) Pain and tenderness localized to the distal two thirds of the medial border of the tibia at the junction of the periosteum and the fascia.

b) Pain in this area, exacerbated by weight bearing or physical activity and relieve by rest.

\section{Exclusion Criteria}

- Stress fracture.

- Subjects with other lower limb musculoskeletal pathology or fractures.

- Open wounds.

- Subjects with muscle herniation, Muscle spasm.

- Subjects with scars which have not yet healed.

- Subjects with compartmental syndrome.

- Parchment -like skin, example in acute episodes of neurodermatits or psoriasis.

- Known allergies to acrylic.

\section{Description of Treatment}

60 subjects were divided into 2 group- Group 1 and Group 2.

\section{Group A}

30 subjects were received advice and education about the treatment. Following this, elastic anti pronation taping technique were be applied on the affected area continuously for three days followed by cryotherapy for 10 minutes. Subjects were be re taped every fourth day for 3 weeks. Subjects were be instructed to remove the tape and tape residue the night prior to attending the testing session. At the end of $1^{\text {st }}$ session the subjects were attend a thirty minute physiotherapy session where advice and education were be reinforced. Regular training were be commenced.

\section{Group B}

30 subjects were received advice and education about the treatment. Following this, space correction taping technique was be applied on the affected area continuously for three days followed by cryotherapy for 10 minutes. Subjects were be re taped every fourth day for 3 weeks. Subjects were instructed to remove the tape and tape residue the night prior to attending the testing session. At the end of $1^{\text {st }}$ session the subjects were attend a thirty minute physiotherapy session where advice and education were be reinforced. Regular training were be commenced.

\section{Results}

Table 1: Normality of pretest and posttest scores ROM and pain in two techniques (Anti pronation taping and Space correction taping) by Kolmogorov Smirnov test

\begin{tabular}{|c|c|c|c|c|c|}
\hline \multirow{2}{*}{ Parameters } & \multirow{2}{*}{ Time points } & \multicolumn{2}{|c|}{ Anti pronation taping } & \multicolumn{2}{c|}{ Space correction taping } \\
\cline { 2 - 6 } & & Z-value & P-value & Z-value & P-value \\
\hline \multirow{3}{*}{ Dorsi Flexion } & Pretest & 1.2080 & 0.1080 & 0.8910 & 0.4060 \\
& Posttest & 1.2970 & 0.0690 & 0.7290 & 0.6620 \\
\cline { 2 - 6 } & Difference & 1.1400 & 0.1480 & 2.0970 & $0.0001^{*}$ \\
\hline \multirow{2}{*}{ Planter Flexion } & Pretest & 1.0290 & 0.2400 & 0.6800 & 0.7450 \\
\cline { 2 - 6 } & Posttest & 1.6100 & $0.0110^{*}$ & 0.7120 & 0.6910 \\
\cline { 2 - 6 } & Difference & 1.0500 & 0.2210 & 1.9080 & $0.0010^{*}$ \\
\hline \multirow{2}{*}{ Pain } & Pretest & 1.3060 & 0.0660 & 1.2930 & 0.0710 \\
\cline { 2 - 6 } & Posttest & 1.3800 & $0.0440^{*}$ & 1.4510 & $0.0300^{*}$ \\
\cline { 2 - 6 } & Difference & 1.3440 & $0.0500^{*}$ & 1.7620 & $0.0040^{*}$ \\
\hline
\end{tabular}

Table 2: Comparison of pretest and posttest Dorsi Flexion scores in two techniques (Anti pronation taping and Space correction taping) by Wilcoxon matched pairs test

\begin{tabular}{|c|c|c|c|c|c|c|c|c|}
\hline Techniques & Time points & Mean & Std.Dv. & Mean Diff. SD Diff. & \% of change & Z-value & P-value \\
\hline \multirow{2}{*}{ Anti pronation taping } & Pretest & 15.10 & 1.84 & & & & & \\
\cline { 2 - 9 } & Posttest & 18.37 & 1.00 & -3.27 & 1.41 & -21.63 & 4.7821 & $0.0001^{*}$ \\
\hline \multirow{2}{*}{ Space correction taping } & Pretest & 14.60 & 1.69 & & & & & \\
\cline { 2 - 9 } & Posttest & 15.40 & 1.54 & -0.80 & 0.61 & -5.48 & 3.9571 & $0.0001^{*}$ \\
\hline
\end{tabular}
$* p<0.05$

Table 3: Comparison of pretest and posttest pain scores in two techniques (Anti pronation taping and Space correction taping) by Wilcoxon matched pairs test

\begin{tabular}{|c|c|c|c|c|c|c|c|c|}
\hline Techniques & Time points & Mean & Std.Dv. & Mean Diff. & SD Diff. & \% of change & Z-value & P-value \\
\hline \multirow{2}{*}{ Anti pronation taping } & Pretest & 6.13 & 0.86 & & & & & \\
\cline { 2 - 9 } & Posttest & 2.87 & 1.11 & 3.27 & 1.31 & 53.26 & 4.6226 & $0.0001 *$ \\
\hline \multirow{2}{*}{ Space correction taping } & Pretest & 6.13 & 0.82 & & & & & \\
\cline { 2 - 9 } & Posttest & 5.27 & 0.83 & 0.87 & 0.78 & 14.13 & 4.0145 & $0.0001 *$ \\
\hline
\end{tabular}




\section{Discussion}

Singh. R, conducted a study on Medial tibia stress syndrome: Who's at risk? A Systematic Review. The purpose of this review is to indentify risk factors in the athletic population for the development of MTSS. The result concluded that there is significant risk factors for MTSS development include high body mass index, female gender, navicular drop, foot pronation, limited hip internal rotation range of motion, increased plantar flexion range of motion, limited straight leg raise, cortical thickness of the tibia, bilateral ankle strength, impaired standing heel-rise test, decreased foot balance, increased miles run per week, less significant exercise history, higher pain level, and longer duration of symptoms ${ }^{[2]}$.

Melanie Franklyn, et al. conducted a study on Aetiology and mechanisms of injury in medial tibia stress syndrome: current and future development. They concluded that MTSS is an overuse fatigue injury involving tibia periostitis in conjunction with cortical bone oedema and microtrauma.The two main mechanisms of injury appear to be a traction-induced periostitis, where the cause is likely to be the soleus and/or the FDL, and micro trauma comprising of oedema and microcracks in the cortical bone which result in debonding of the osteons and subcutaneous periostitis on the surface of the tibia ${ }^{[7]}$.

Merinus Winters, et al. conducted a study on treatment of medial tibia stress syndrome: a systemic review to assess the effectiveness of any intervention in the treatment of MTSS. They concluded that there are studies suggesting that iontophoresis, phonophoresis, ice massage, ultrasound, periosteal pecking and extracorporeal shockwave therapy are effective. None of the studies are sufficiently free from methodological bias to recommend any of the treatments investigated ${ }^{[4]}$.

Hylton B. Menz, et al. conducted a study on Planus Foot Posture and Pronated Foot Function are Associated with Foot Pain: The Framingham Foot Study. To examine the associations of foot posture and foot function to foot pain. They found that both planus foot posture and pronated foot function are associated with foot pain. Specifically, planus foot posture was found to be associated with arch pain and pronated foot function was significantly associated with generalized foot pain and heel pain in men ${ }^{[8]}$.

Maarten Hendrik Moen, et al. conducted a study on the treatment of medial tibia stress syndrome in athletes; a randomized clinical trial. 74 athletes were included and randomized to three treatment groups. Group one performed a graded running program, group two performed a graded running program with additional stretching and strengthening exercises for the calves, while group three performed a graded running program with an additional sports compression stocking. The primary outcome measure was: time to complete a running program and secondary outcome was: general satisfaction with treatment. No significant differences were found between the three treatment groups in days to complete a running program and satisfaction with the treatment. The study does provide insight in recovery of MTSS in athletes with an average time to complete a running program ${ }^{[9]}$.

R. Michael Galbraith, et al. conducted a study on Medial tibia stress syndrome: conservative treatment option. The purpose of this article is to review published literature regarding conservative treatment options for MTSS and provide recommendations for sports medicine clinicians for improved treatment and patients outcomes. They concluded that most studies support rest, ice, and analgesics in the acute phase. Many experts also recommend modifying the training routine, stretching, and strengthening the lower extremity, wearing appropriate footwear, using orthotics and manual therapy to correct biomechanical abnormalities, and gradually return to activity ${ }^{[5]}$.

\section{References}

1. Franklyn M, Oakes B. Aetiology and mechanisms of injury in medial tibia stress syndrome: Current and future developments. World journal of orthopedics. 2015; 6(8):577.

2. Singh R. Medial Tibia Stress Syndrome: Who's at risk? A systematic Review. J Phys Med Rehabil Disabil. 2016; 2:014.

3. Yates $\mathrm{B}$, White $\mathrm{S}$. The incidence and risk factors in the development of medial tibia stress syndrome among naval recruits. The American journal of sports medicine. 2004; 32(3):772-80.

4. Winters M, Eskes M, Weir A, Moen MH, Backx FJ, Bakker EW. Treatment of medial tibia stress syndrome: a systematic review. Sports Medicine. 2013; 43(12):1315-33.

5. Galbraith RM, Lavallee ME. Medial tibia stress syndrome: conservative treatment options. Current reviews in musculoskeletal medicine. 2009; 2(3):127-33.

6. Prusak KM, Prusak KA, Hunter I, Seeley MK, Hopkins JT. Comparison of Two Taping Techniques on Navicular Drop and Center-of-Pressure Measurements During Stance. Athletic Training and Sports Health Care. 2014; 6(6):25260.

7. Melanie Franklyn and Barry Oakes. Aetiology and mechanisms of injury in medial tibia stress syndrome: Current and future developments. World J Orthop. 2015; 6(8):577-589.

8. Menz HB, Dufour AB, Riskowski JL, Hillstrom HJ, Hannan MT. Association of planus foot posture and pronated foot function with foot pain: the Framingham foot study. Arthritis care \& research. 2013; 65(12):1991-9.

9. Moen MH, Holtslag L, Bakker E, Barten C, Weir A, Tol JL, et al. The treatment of medial tibia stress syndrome in athletes; a randomized clinical trial. Sports Medicine, Arthroscopy, Rehabilitation, Therapy \& Technology. 2012; 4(1): 12 . 